\title{
A Novel Location-Awareness Method Using CubeSats for Locating the Spot Beam Emitters of Geostationary Communications Satellites
}

\author{
Weicai Yang $\mathbb{D}^{\mathrm{D}},{ }^{1}$ Qing Chang, ${ }^{1}$ Allison Kealy, ${ }^{2}$ Yong Xu ${ }^{D},{ }^{1}$ and Tianyi Lan ${ }^{3}$ \\ ${ }^{1}$ School of Electronic and Information Engineering, Beihang University, Beijing 100191, China \\ ${ }^{2}$ School of Science, RMIT University, Melbourne, VIC, Australia \\ ${ }^{3}$ SpaceKey Technology, Ltd., Beijing 100191, China \\ Correspondence should be addressed to Yong Xu; xuyong1518@163.com
}

Received 21 August 2017; Accepted 20 December 2017; Published 22 January 2018

Academic Editor: Nazrul Islam

Copyright (C) 2018 Weicai Yang et al. This is an open access article distributed under the Creative Commons Attribution License, which permits unrestricted use, distribution, and reproduction in any medium, provided the original work is properly cited.

\begin{abstract}
As more spacecraft are launched into the Geostationary Earth Orbit (GEO) belt, the possibility of fatal collisions or unnecessary interference between spacecraft increases. In this paper, a new location-awareness method that uses CubeSats is proposed to assist with radiofrequency (RF) domain verification by means of awareness and identification of the positions of the spot beam emitters of communications satellites in geostationary orbit. By flying a CubeSat (or a constellation of CubeSats) through the coverage area of a spot beam, the spot beam emitter's position is identified and the spot beam's pattern knowledge is characterized. The geometry, the equations of motion of the spacecraft, the measurement process, and the filtering equations in a location system are addressed with respect to the location methods investigated in this study. A realistic scenario in which a CubeSat receives signals from GEO communications satellites is simulated using the Systems Tool Kit (STK). The results of the simulation and the analysis presented in this study provide a thorough verification of the performance of the location-awareness method.
\end{abstract}

\section{Introduction}

As space becomes an increasingly congested, contested, and competitive environment, the need for enhanced space-based capabilities only increases over time [1]. As more spacecraft are launched into the Geostationary Earth Orbit (GEO) belt, the possibility of fatal collisions or unnecessary interference between spacecraft increases [2]. This interference and additional congestion include the radiofrequency (RF) domain, with global satellite communications taking advantage of numerous and ever-increasing number of spot beam emitters with varying frequencies and pointing locations [3]. Therefore, building a global RF beam pattern database should help mitigate global RF interference by offering the best knowledge of the positions of spacecraft source emitters and mapping the pattern knowledge of the spot beams of communications satellites in or near geostationary orbit. An awareness and identification method can provide reasonable estimates of beam location, gain, frequency, and emitter position that are useful for verifying or monitoring a spot beam's pattern. The ability of this method to identify the location of spot beam emitters through the use of nanosatellite-class (i.e., CubeSat) spacecraft is analyzed.

To verify and monitor the global RF signal usage of satellites in the GEO belt, the traditional approach takes advantage of a wideband spectrum receiver at a fixed ground station terminal [4]. Although satellite signals can be measured in this manner, the ground data obtained via the above measurements represent a single ground point in a region and are applicable only in particular area. To circumvent these difficulties, a CubeSat mission was recently proposed as an effective method of verifying the spot beams of geostationary communications satellites. The CubeSat mission would carry out this verification by mapping GEO spot beams from low Earth orbit (LEO), making it possible to establish a monitoring station with no territorial limitation and to generate a beam pattern database with higher resolution than one based on fixed ground site measurements of GEO signals [5]. 
Unfortunately, this mission did not provide an appropriate method for identifying the location of a spot beam emitter, resulting in the database containing incomplete RF knowledge. Based on the above, it is necessary to put forward a location-awareness method to identify the positions of spot beam emitters and obtain complete RF knowledge with respect to geostationary communications satellites.

In another study, Bentley investigated RF signal geolocation techniques applied to geostationary satellites. This technique focused on the use of time difference of arrival (TDOA) and frequency difference of arrival (FDOA) to locate interference [6]. Small studied multiple methods of locating source transmitters on the ground and simulated different methods with various sensor configurations and signal properties [7]. However, the aforementioned methods are useful only for locating ground emitters. Moreover, multiple monitoring satellites are required to extract meaningful geolocation information at the same time. It is difficult to determine the locations of the spot beam emitters of geostationary communications satellites because the spot beam is very narrow. In this case, the spot beam cannot cover multiple monitoring satellites at the same time. Research on methods of determining a transmitter's position with a single moving sensor has also recently gained interest. The bearingonly method is an effective solution for determining the angle of arrival (AOA) from a signal source to a moving sensor to estimate the position of a signal emitter [8-12]. Unfortunately, earlier location-based methods for measuring bearings using a single observer were applicable only to ground and air targets. Since the target and observer are both satellites, with kinetic characteristics that are different from those of ground and air objects, the above methods do not work in the spot beam emitter location-awareness method that uses a CubeSat $[13,14]$.

The shortcomings of the above processes, which are currently used to identify the emitter locations of GEO communications satellites, call for a more active and robust GEO spot beam emitter location-awareness method. In this paper, we propose a new location-awareness method that uses a CubeSat to locate the spot beam emitters of geostationary communications satellites. Section 2 presents the problem formulation. Section 3 focuses on simulations of the CubeSat location-awareness process. In Section 4 , the different metrics used to evaluate the performances of algorithms are discussed. Finally, concluding remarks are provided in Section 5.

\section{Problem Formulation}

In this section, first, the geometry used in the spot beam emitter location awareness is described. Then, the spacecraft dynamics and the measurement process associated with the onboard sensor are discussed. Finally, the extended Kalman filter (EKF) estimation algorithm is applied to estimate the position and velocity of a GEO communications satellite's emitter.

2.1. Geometry for Spot Beam Emitter Location Awareness. In this paper, a novel approach that involves flying a CubeSat through spot beams of an emitter at the altitude of an LEO

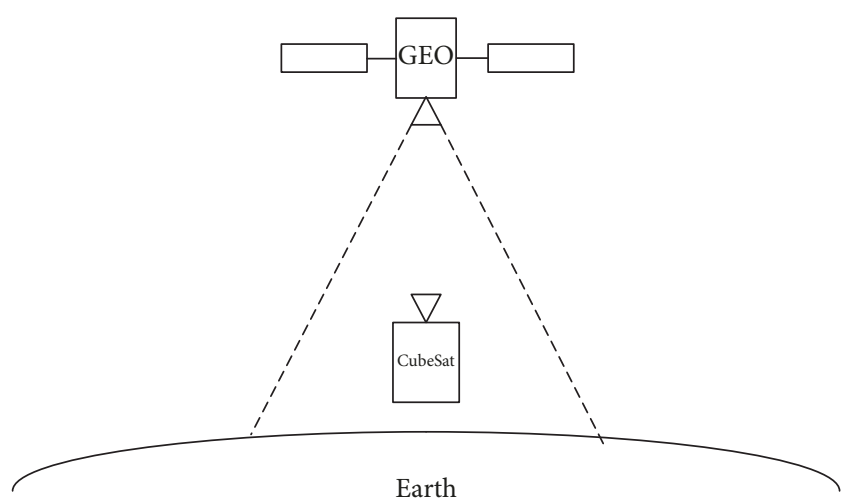

Figure 1: Awareness method of locating a GEO signal emitter.

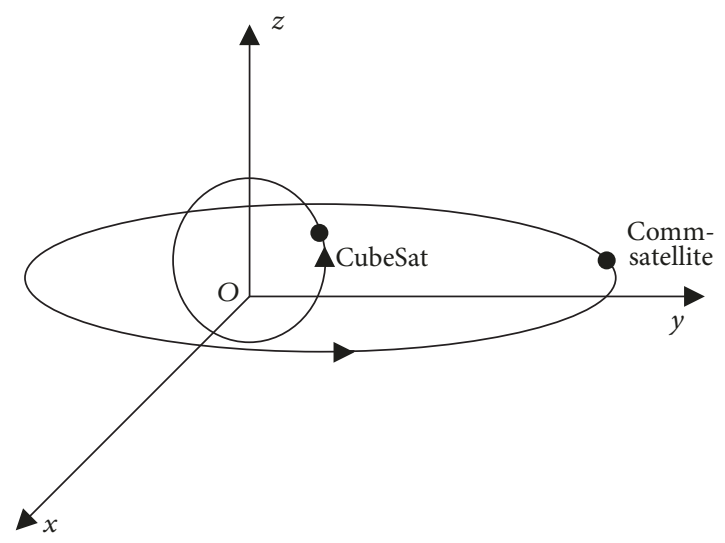

FIGURE 2: Geometry of the location-awareness system.

is adopted to identify the location of a spot beam emitter. Figure 1 depicts the awareness method of locating a GEO signal emitter.

The model for the location-awareness system is set up in the standard J2000 Earth centered inertial (ECI) reference frame. The communications satellite and the CubeSat are regarded as two particles that move in response to Earth's gravity. The details are given in Figure 2.

In the J2000 ECI reference frame's coordinates, the position and velocity vectors for the communications satellite are expressed as $r_{\text {com }}=\left(x_{\text {com }}, y_{\text {com }}, z_{\text {com }}\right)$ and $v_{\text {com }}=$ $\left(v_{x \mathrm{com}}, v_{y \text { com }}, v_{z \mathrm{com}}\right)$, respectively. The corresponding vectors for the CubeSat are expressed as $r_{\text {cub }}=\left(x_{\text {cub }}, y_{\text {cub }}, z_{\text {cub }}\right)$ and $v_{\text {cub }}=\left(v_{x \text { cub }}, v_{y \text { cub }}, v_{z \text { cub }}\right)$.

2.2. Equations of Motion for the Spacecraft. Normally, we assume that Earth and a satellite can be considered a sphere and a particle, respectively, and that they constitute a twobody system. In the two-body system, the satellite is affected by Earth's gravity. The gravity $(F)$ can be written as

$$
F=-\frac{G M m}{r^{2}} \cdot \frac{\mathbf{r}}{r}
$$

where $G$ is the gravitational constant, $M$ and $m$ denote the masses of Earth and the satellite, respectively, $\mathbf{r}=(x, y, z)$ is 
the satellite's position vector, and $r$ is a scalar that denotes the distance between the satellite and the center of Earth.

According to Newton's second law, the satellite's motion is expressed as follows:

$$
\mathbf{a}=\frac{F}{m}=-\frac{\mu}{r^{2}} \cdot \frac{\mathbf{r}}{r},
$$

where $\mathbf{a}=\left(a_{x}, a_{y}, a_{z}\right)$ is the three-dimensional acceleration vector of the satellite and $\mu=G M$ is the geocentric gravitational constant, the value of which is approximately equal to $3.986004415 \times 10^{14} \mathrm{~m}^{3} / \mathrm{s}^{2}$, according the International Astronomical Union (IAU).

The parameters of the spacecraft's motion at time $t=$ $i T, i=1,2,3, \ldots$ ( $T$ denotes the sampling interval) can be calculated using the following iterative method:

$$
\begin{aligned}
& \mathbf{r}_{i}=\mathbf{r}_{i-1}+\mathbf{v}_{i-1} \cdot T+\mathbf{a}_{i-1} \cdot \frac{T^{2}}{2} \\
& \mathbf{v}_{i}=\mathbf{v}_{i-1}+\mathbf{a}_{i-1} \cdot T \\
& \mathbf{a}_{i}=-\frac{\mu}{r^{2}} \cdot \frac{\mathbf{r}_{i}}{r} .
\end{aligned}
$$

Furthermore, the state vector of the spacecraft's motion can be derived as follows:

$$
\mathbf{X}_{i}=\mathbf{X}_{i-1}+\dot{\mathbf{X}}_{i-1} \cdot T+B \cdot \dot{\mathbf{X}}_{i-1} \cdot \frac{T^{2}}{2}
$$

where $\mathbf{X}=\left[\begin{array}{llllll}r_{x} & r_{y} & r_{z} & v_{x} & v_{y} & v_{z}\end{array}\right]^{\prime}$ is a six-dimensional state vector that describes the position and velocity of the spacecraft, with the "prime" symbol (') denoting matrix transposition, $\dot{\mathbf{X}}=\left[\begin{array}{llllll}v_{x} & v_{y} & v_{z} & a_{x} & a_{y} & a_{z}\end{array}\right]^{\prime}$ is the derivative of $\mathbf{X}$, and

$$
B=\left[\begin{array}{ll}
O & I \\
O & O
\end{array}\right],
$$

where $I$ is a $3 \times 3$ identity matrix and $O$ is a $3 \times 3$ null matrix.

In the two-body problem, substituting (2) into (4) yields the expression for the state model of the spacecraft:

$\mathrm{X}_{i}$

$$
\left[\begin{array}{cccccc}
1-\frac{\mu}{r^{3}} \cdot \frac{T^{2}}{2} & 0 & 0 & T & 0 & 0 \\
0 & 1-\frac{\mu}{r^{3}} \cdot \frac{T^{2}}{2} & 0 & 0 & T & 0 \\
0 & 0 & 1-\frac{\mu}{r^{3}} \cdot \frac{T^{2}}{2} & 0 & 0 & T \\
-\frac{\mu}{r^{3}} \cdot T & 0 & 0 & 1 & 0 & 0 \\
0 & -\frac{\mu}{r^{3}} \cdot T & 0 & 0 & 1 & 0 \\
0 & 0 & -\frac{\mu}{r^{3}} \cdot T & 0 & 0 & 1
\end{array}\right] \mathbf{X}_{i-1}
$$

$$
=A_{i-1} \mathbf{X}_{i-1} \text {. }
$$

It should be noted that (6) is derived with the assumption that the spacecraft's acceleration is constant between the two samplings. This assumption is discussed in Section 4.

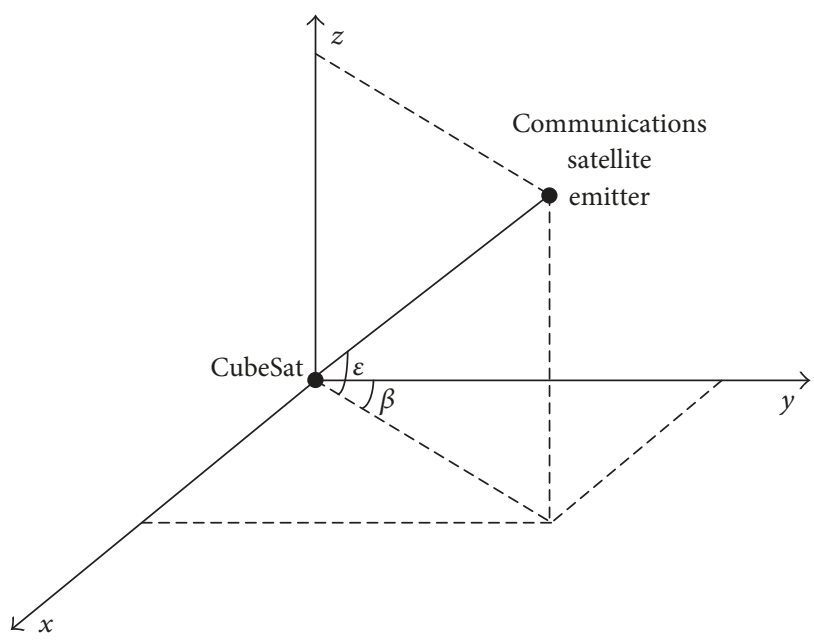

FIGURE 3: Overall emitter and CubeSat geometry showing azimuth and elevation angles.

2.3. Measurement Process. To obtain a measurement for the spot beam emitter of a geostationary communications satellite, it is necessary to have a bearing sensor onboard the CubeSat and to obtain the position information of the CubeSat. The location-aware CubeSat, during a notional orbit, is expected to physically fly through a large number of spot beams with coverage areas of various sizes. Once the emitter signal is detected, the CubeSat with a bearing sensor measures azimuth and elevation angles of the incoming signals and outputs position information at the same time. The position of the CubeSat can be obtained from the GPS receiver mounted on it [15]. Figure 3 shows the overall azimuth and elevation angles of the CubeSat with respect to the target emitter. The angles are given by

$$
\begin{aligned}
& \beta=\tan ^{-1}\left(\frac{x_{\mathrm{com}}-x_{\mathrm{cub}}}{y_{\mathrm{com}}-y_{\mathrm{cub}}}\right) \\
& \varepsilon=\tan ^{-1}\left(\frac{z_{\mathrm{com}}-z_{\mathrm{cub}}}{\sqrt{\left(x_{\mathrm{com}}-x_{\mathrm{cub}}\right)^{2}+\left(y_{\mathrm{com}}-y_{\mathrm{cub}}\right)^{2}}}\right) .
\end{aligned}
$$

It should be noted that (7) are defined in the CubeSat frame. The CubeSat frame's axes are parallel to Earth Centered Inertial (ECI) frame.

Furthermore, the measurement matrix defined by (8) is a nonlinear problem:

$$
Z=\left[\begin{array}{l}
\beta \\
\varepsilon
\end{array}\right]=f(\mathbf{X})
$$

2.4. Description of the Extended Kalman Filter Equations. The state transition vector is given by

$$
\widehat{\mathbf{X}}(i)=A(i-1) \mathbf{X}(i-1)+\mathbf{W}(i),
$$

where $\widehat{\mathbf{X}}(i)$ represents a filter estimate of the true state vector $\mathbf{X}(i)$ based on $i$ measurements and the error sequences 
described by $\mathbf{W}(i)$ with variance $Q=10^{-2} \mathrm{~m}^{2} / \mathrm{s}^{4}$. It should be noted that $\mathbf{W}(i)$ is introduced to model the disturbance on satellite movement.

The corresponding filter estimate of the state error covariance is given by

$$
\widehat{P}(i)=A(i-1) P(i-1) A^{\prime}(i-1)+Q .
$$

The nonlinear measurement equations are rewritten with additive measurement noise as

$$
\begin{aligned}
\beta= & \tan ^{-1}\left(\frac{x_{\mathrm{com}}-x_{\mathrm{cub}}}{y_{\mathrm{com}}-y_{\mathrm{cub}}}\right)+\delta_{\beta} \\
\varepsilon= & \tan ^{-1}\left(\frac{z_{\mathrm{com}}-z_{\mathrm{cub}}}{\sqrt{\left(x_{\mathrm{com}}-x_{\mathrm{cub}}\right)^{2}+\left(y_{\mathrm{com}}-y_{\mathrm{cub}}\right)^{2}}}\right) \\
& +\delta_{\varepsilon},
\end{aligned}
$$

where $\delta_{\beta}$ and $\delta_{\varepsilon}$ are assumed to be mutually independent zero-mean Gaussian white noise with variances $R$.

Computing the partial derivative of nonlinear equations (11) yields

$$
\begin{aligned}
& \frac{\partial \beta}{\partial x_{\mathrm{com}}} \\
& =\frac{\left(y_{\mathrm{com}}-y_{\mathrm{cub}}\right)^{2}}{\left(x_{\mathrm{com}}-x_{\mathrm{cub}}\right)^{2}+\left(y_{\mathrm{com}}-y_{\mathrm{cub}}\right)^{2}} \cdot \frac{1}{y_{\mathrm{com}}-y_{\mathrm{cub}}} \\
& \frac{\partial \beta}{\partial y_{\mathrm{com}}}=\frac{x_{\mathrm{cub}}-x_{\mathrm{com}}}{\left(x_{\mathrm{com}}-x_{\mathrm{cub}}\right)^{2}+\left(y_{\mathrm{com}}-y_{\mathrm{cub}}\right)^{2}} \\
& \frac{\partial \beta}{\partial z_{\mathrm{com}}}=0 \\
& \frac{\partial \varepsilon}{\partial y_{\mathrm{com}}} \\
& =\frac{\left(z_{\mathrm{com}}-z_{\mathrm{cub}}\right) \cdot\left(x_{\mathrm{cub}}-x_{\mathrm{com}}\right)}{\left(x_{\mathrm{com}}-x_{\mathrm{cub}}\right)^{2}+\left(y_{\mathrm{com}}-y_{\mathrm{cub}}\right)^{2}+\left(z_{\mathrm{com}}-z_{\mathrm{cub}}\right)^{2}} \\
& \quad \frac{1}{\sqrt{\left(x_{\mathrm{com}}-x_{\mathrm{cub}}\right)^{2}+\left(y_{\mathrm{com}}-y_{\mathrm{cub}}\right)^{2}}} \\
& \frac{\partial \varepsilon}{\partial y_{\mathrm{com}}} \\
& =\frac{\left(z_{\mathrm{com}}-z_{\mathrm{cub}}\right) \cdot\left(y_{\mathrm{cub}}-y_{\mathrm{com}}\right)}{\left(x_{\mathrm{com}}-x_{\mathrm{cub}}\right)^{2}+\left(y_{\mathrm{com}}-y_{\mathrm{cub}}\right)^{2}+\left(z_{\mathrm{com}}-z_{\mathrm{cub}}\right)^{2}} \\
& \quad \cdot \frac{1}{\sqrt{\left(x_{\mathrm{com}}-x_{\mathrm{cub}}\right)^{2}+\left(y_{\mathrm{com}}-y_{\mathrm{cub}}\right)^{2}}} \\
& \frac{\partial \varepsilon}{\partial z_{\mathrm{com}}}
\end{aligned}
$$

The linearized form of the nonlinear measurement matrix becomes

$$
\begin{aligned}
H(i) & =\left.\frac{\partial f}{\partial \mathbf{X}}\right|_{\mathbf{X}=\widehat{\mathbf{x}}(\mathbf{i})} \\
& =\left[\begin{array}{llllll}
\frac{\partial \beta}{\partial x_{\text {com }}} & \frac{\partial \beta}{\partial y_{\text {com }}} & \frac{\partial \beta}{\partial z_{\text {com }}} & 0 & 0 & 0 \\
\frac{\partial \varepsilon}{\partial x_{\text {com }}} & \frac{\partial \varepsilon}{\partial y_{\text {com }}} & \frac{\partial \varepsilon}{\partial z_{\text {com }}} & 0 & 0 & 0
\end{array}\right] .
\end{aligned}
$$

The Kalman gain can be calculated as follows:

$$
K(i)=P(i) H^{\prime}(i)[H(i) P(i) H(i)+R(i)]^{-1} .
$$

The state estimate and error covariance are updated using (15), respectively.

$$
\begin{aligned}
\mathbf{X}(i) & =\widehat{\mathbf{X}}(i)+K(i)[z(i)-f(\widehat{\mathbf{X}}(i))] \\
P(i) & =[I-K(i) H(i)] \widehat{P}(i) .
\end{aligned}
$$

\section{Model Simulation}

The CubeSat location-awareness process was modeled using the Systems Tool Kit (STK) to simulate a scene in which a CubeSat receives signals from GEO communications satellites, and bearing data and the CubeSat's position can be obtained from it. The Intelsat Galaxy 28 (G-28) GEO satellite was chosen for modeling in the Ku-band. The G-28 satellite maintains spot beams within the Ku-band and the C-band [16], but these were not jointly modeled since the Ku-band beams, being smaller, provided a better means for capability assessment. It was determined that if the CubeSat could reliably locate the Ku-band beam emitter, then the C-band beam emitter for larger coverage could also be located.

Since most companies do not publish exact antenna and transmission power levels due to their proprietary nature, typical antenna sizes for the simulated K-band beams were assumed in this paper [17-19]. The simulated Ku-band antennas were assumed to be $1 \mathrm{~m}$ in diameter and beams were set to approximately $12 \mathrm{GHz}$ [20]. To model the beams accurately, half-power sensors were combined to notionally match the conical shape of the half-power beam width (HPBW) of the patterns. Figure 4 shows an Earth view of the modeled G-28 emitter of beams in the Ku-band.

A special case beam used the same antenna size as that of the Ku-band beams but had a frequency that was increased from the base $\mathrm{Ku}$-band $(12 \mathrm{GHz})$ to the Ka-band $(30 \mathrm{GHz})$. Increasing the transmission frequency reduced the HPBW and thus reduced the beam's coverage area [20]. The increased-frequency emitter, therefore, became harder to locate since it required a much shorter time than lowerfrequency emitter. The model of the Ka-band beams is shown in Figure 5.

Using G-28 as the basic GEO communications satellite for the CubeSat location-awareness scenario, a CubeSat was then added to the scenario as the monitoring satellite. Note that the CubeSat location-awareness model was built based on the fact that GEO satellite beams can be detected and mapped under 


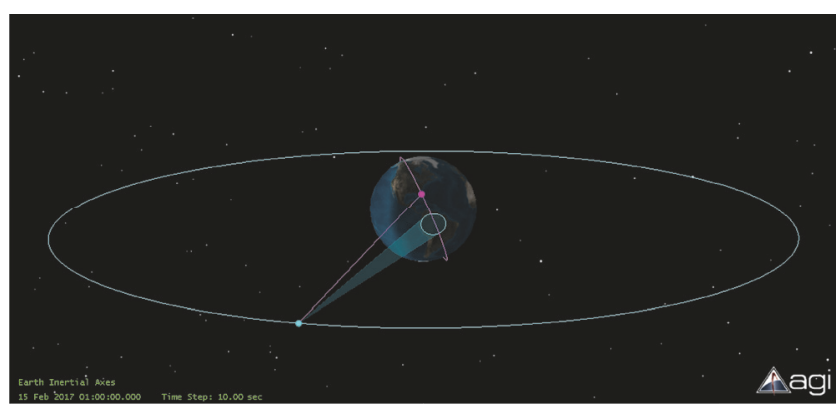

FIGURE 4: Modeled G-28 emitter of beams in the Ku-band.

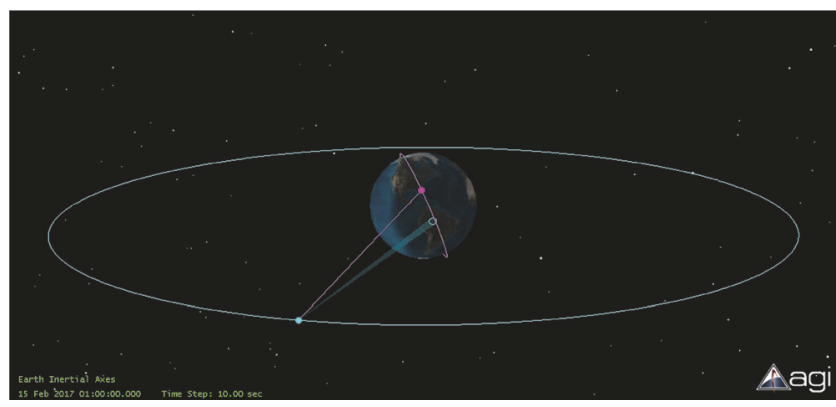

Figure 5: Modeled G-28 emitter of beams in the Ka-band.

TABLE 1: Constraints for the CubeSat constellations and orbits.

\begin{tabular}{lc}
\hline Constellation altitude: & $350 \mathrm{~km}-500 \mathrm{~km}$ \\
Constellation inclination: & 68 degrees \\
Payload sampling rate: & 1 s per data point \\
CubeSat planes: & 2 planes \\
Number of CubeSats: & 3 satellites \\
Duration: & 3 days \\
\hline
\end{tabular}

the best monitoring conditions. This is because the best beam pattern knowledge can be gained. Therefore, it is necessary to satisfy the basic constraints on CubeSat constellations and orbits mentioned in another paper [5]. These constraints are summarized in Table 1 . It must be noted that multiple CubeSats were modeled to increase the responsiveness and shorten the necessary data collection duration of the project. However, in the location-awareness process, any CubeSat can locate a GEO emitter alone. Furthermore, further research is still a matter of concern for the CubeSat constellations and orbits. A heuristic design technique was recommended in paper [21].

After modeling the CubeSat location awareness, the positions of the CubeSat and the GEO satellite were collected whenever the CubeSat received a signal from the GEO emitter. Finally, this position information was converted into bearing measurement data and then exported to MATLAB for verification of the location algorithm.

\section{Simulation Results and Analysis}

4.1. Simulation of the CubeSat Location-Awareness Geometry. The CubeSat location-awareness model was run for three

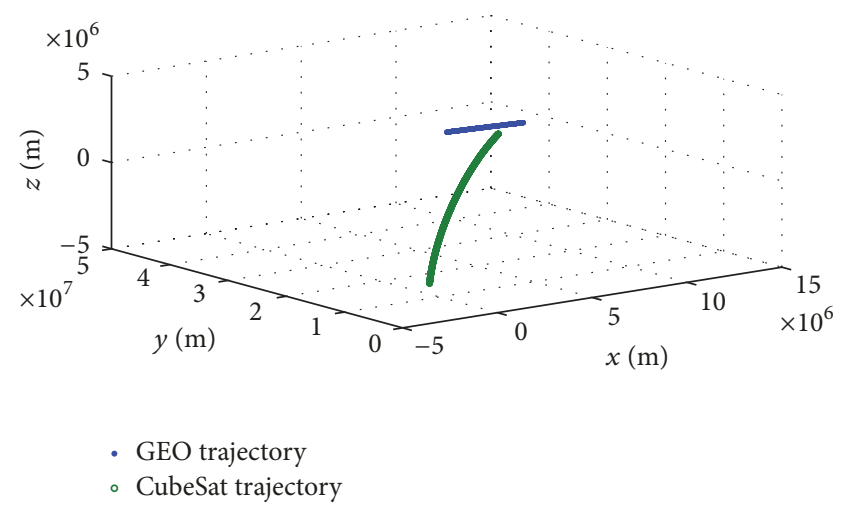

Figure 6: CubeSat and GEO satellite geometry.

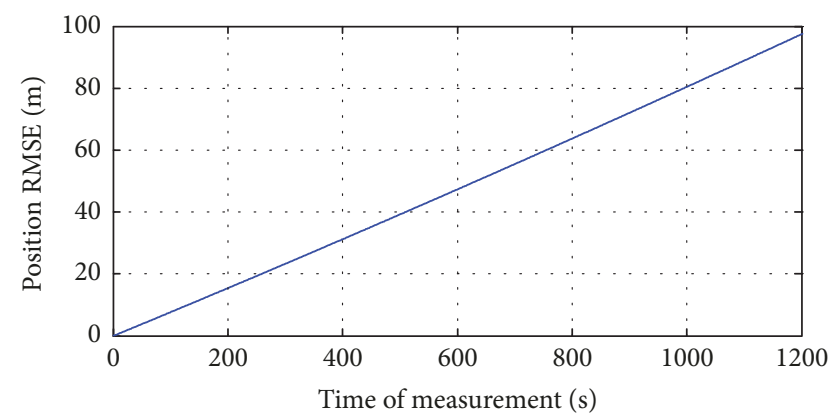

Figure 7: State model error.

days using the STK. The simulation results indicated that the CubeSat received a signal from the GEO satellite many times and that the duration of each signal reception was less than 1200 s. A set of data for the CubeSat and the GEO satellite geometry was chosen for the analysis of the final positioning results, shown in Figure 6.

As discussed in the previous section, the satellite's motion is complex and nonlinear. As an approximation method, we considered the two-body problem with constant acceleration between two samplings. However, this approximation method could produce position errors in the locationawareness process. The errors were calculated by comparing the state model established by (4) with the GEO satellite's real trajectory simulated by STK. In Figure 7, the position error is graphed against the time of measurement. The largest position error with respect to the simulation result was $98 \mathrm{~m}$.

4.2. Simulation of the Ku-Band Beam Emitter. To check the performance of the EKF, the algorithm was tested in three different cases for the Ku-band beam model. The simulation time was set to $1200 \mathrm{~s}$, and the filtering cycle was $1 \mathrm{~s}$ long. The initial position error was fixed at $10^{6} \mathrm{~m}$ in the first case. To simulate the data measurement errors, the raw bearings were corrupted by additive zero-mean Gaussian noise prior to processing. Three different noise levels were used to analyze the position and velocity errors. Figure 8 depicts the root mean square error (RMSE) in the position and the velocity obtained with a root mean square (RMS) noise level of $0.01^{\circ}$. Figures 9 and 10 correspond to RMS noise levels of $0.05^{\circ}$ and 

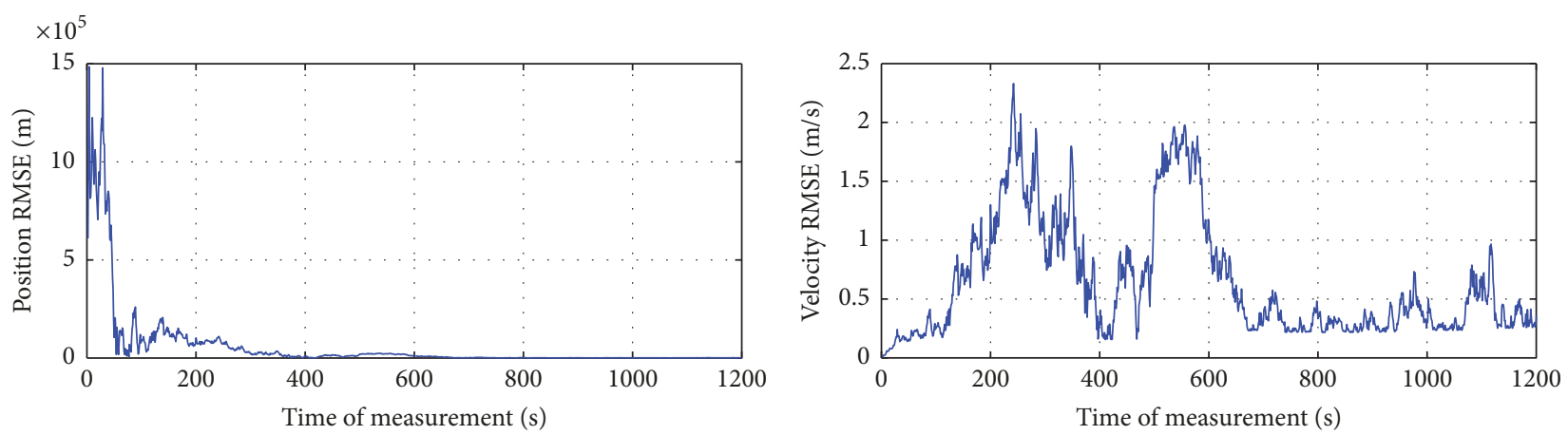

FIGURE 8: RMSEs of the position and velocity for a measurement noise level of $0.01^{\circ}$.
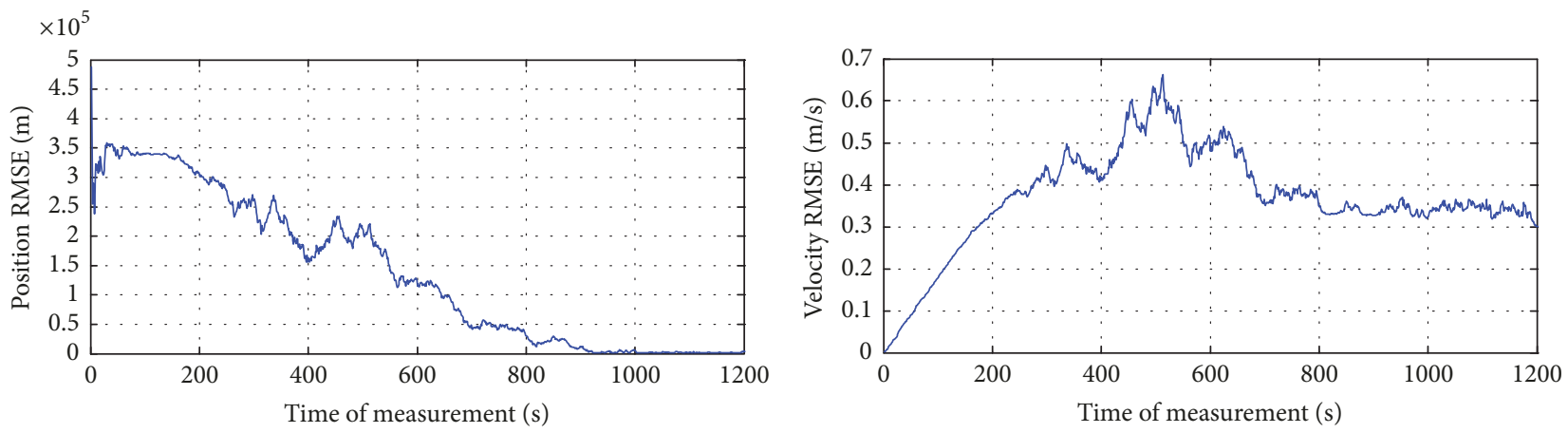

FigURE 9: RMSEs of the position and velocity for a measurement noise level of $0.05^{\circ}$.
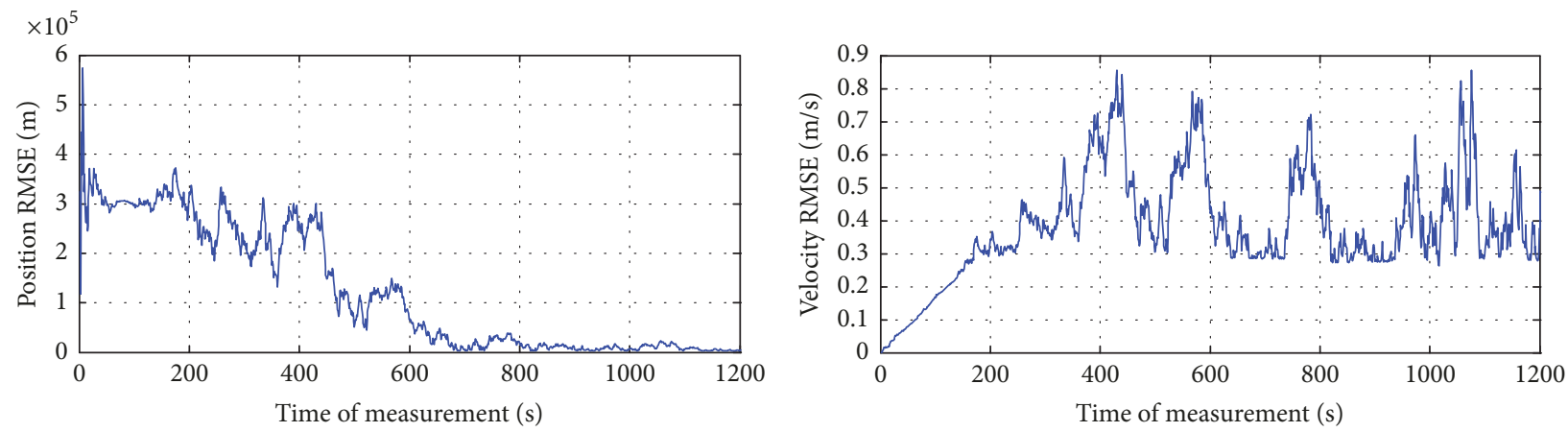

FIGURE 10: RMSEs of the position and velocity for a measurement noise level of $0.1^{\circ}$.

$0.1^{\circ}$, respectively. The measurement accuracy can be met by direction finding algorithm in paper [22].

Figures 8-10 show that, in the location-awareness model, the EKF is able to successfully estimate the position of the GEO satellite emitter and subsequently continue to track it. The increase in the noise level decreases the time required for convergence and results in a smaller error magnitude when convergence is achieved. When the measurement noise level is $0.1^{\circ}$, the solution does not really converge until the simulation is nearly complete, that is, after 926 samplings. When the measurement noise level is $0.01^{\circ}$, the solution converges when the simulation is approximately halfway complete, that is, after approximately 650 measurements. The simulation results for different noise levels are summarized in Table 2. As a result, the bearing measurement must be accurate to within
TABLE 2: Simulation results for the different noise levels.

\begin{tabular}{lcc}
\hline Noise level, ${ }^{\circ}$ & RMSE convergence value, $\mathrm{m}$ & Convergence time, $\mathrm{s}$ \\
\hline 0.01 & 962 & 672 \\
0.05 & 2126 & 923 \\
0.1 & 8294 & 926 \\
\hline
\end{tabular}

$0.01^{\circ}$ to satisfy the performance requirement of the locationawareness method. Moreover, the RMSE of the velocity is a relatively stable estimate, as indicated by Figures 8-10.

In the second case, the initial position error was simulated with the maximum value $\left(10^{7} \mathrm{~m}\right)$, and the measurement noise level was set to $0.01^{\circ}$. Figure 11 depicts the RMSEs of the 

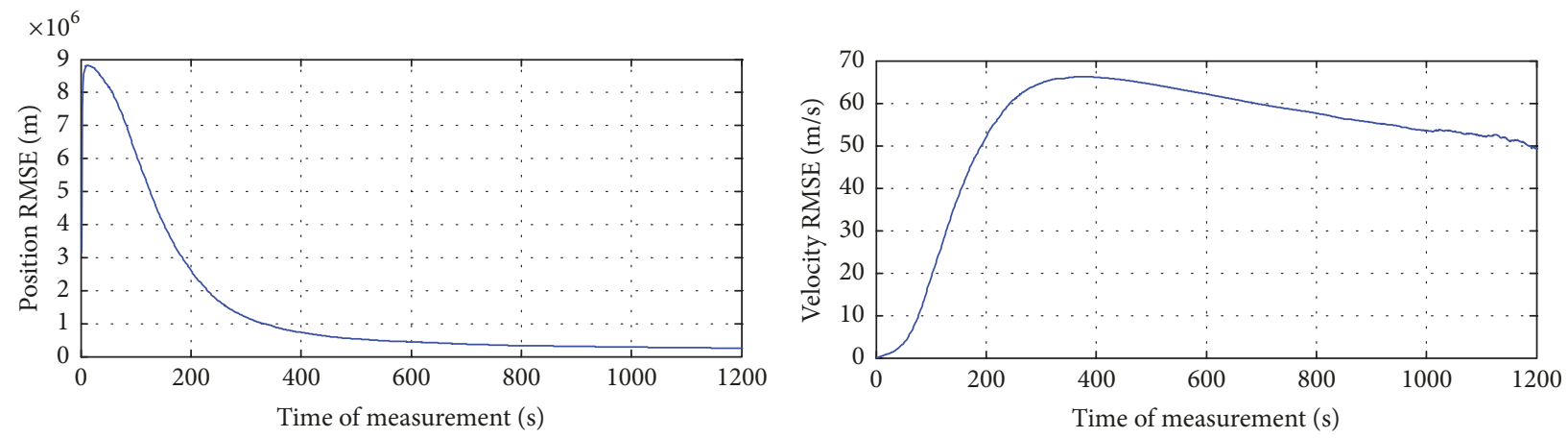

FIGURE 11: RMSEs of the position and velocity in the second case.
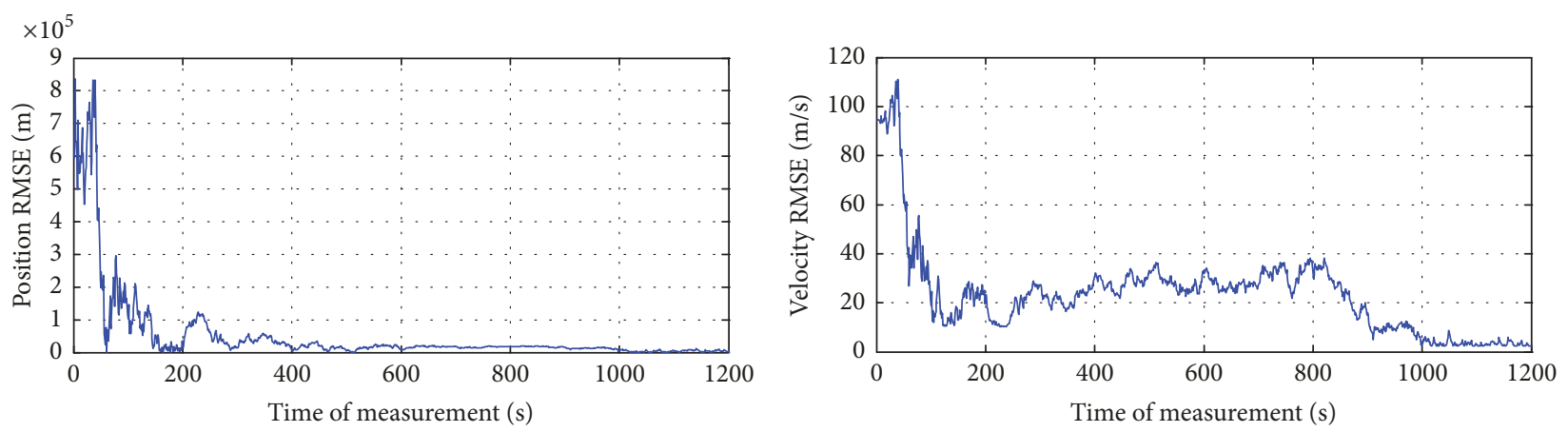

FIgURE 12: RMSEs of the position and velocity in the third case.
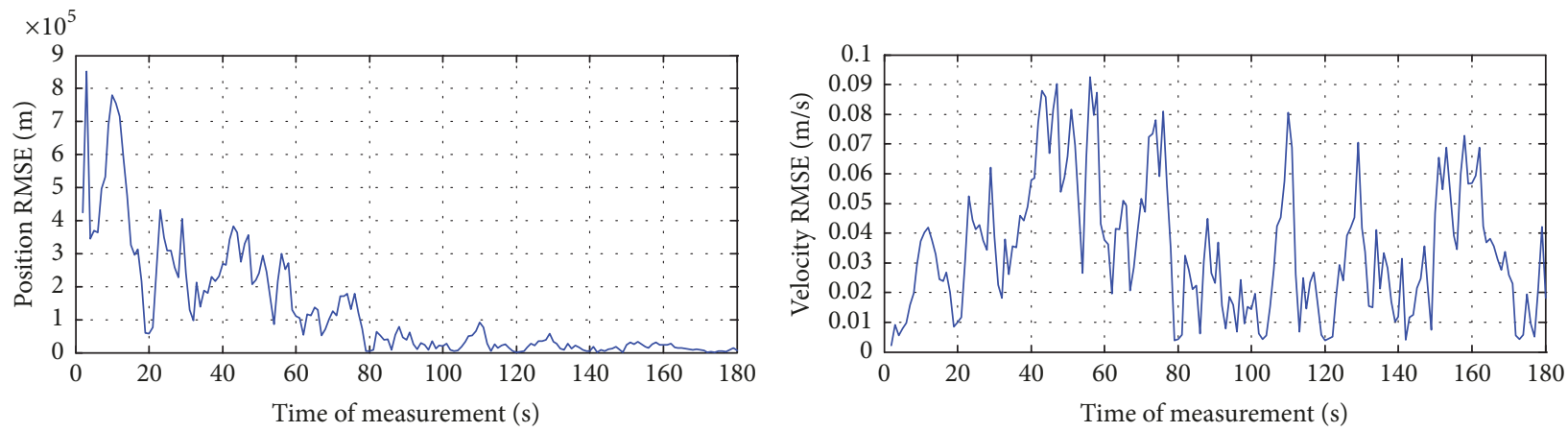

FIGURE 13: RMSEs of the position and velocity for the Ka-band beam emitter.

position and velocity with respect to time. It shows that the performance of the EKF is significantly worse when the initial condition is poor.

In the third case, an initial error of $100 \mathrm{~m} / \mathrm{s}$ in the velocity was added to the simulation. The initial position error was set to $10^{6} \mathrm{~m}$, and the simulated measurement noise level was $0.01^{\circ}$. The RMSEs of the position and velocity are depicted in Figure 12.

As in the previous results, the RMSEs of the position and velocity converge to steady-state values as the time of measurement increases. However, a longer time is required for convergence, and a greater error magnitude is found when the initial error in the velocity is added to the simulation. Furthermore, the results presented here demonstrate that the performance of the location-awareness method is affected by the initial state error. As a result, further improvements to the algorithms will be made to reduce the influence of the initial state error and enhance the convergence speed.

4.3. Simulation of Ka-Band Beam Emitter. The time required for the CubeSat to receive a Ka-band beam emitter signal is less than $180 \mathrm{~s}$, according to the location-awareness model result obtained using the STK. The simulation data for the Ka-band beam emitter were output and then simulated using MATLAB. In this simulation, the initial position error was $10^{6} \mathrm{~m}$. In Figure 13, the RMSEs of the position and velocity as functions of the measurement time are shown. By the 172nd computation, the RMSE of the position had settled from the initial choice of $10^{6} \mathrm{~m}$ to approximately $5 \times 10^{3} \mathrm{~m}$. This 
result is worse because the time required to receive a signal is much shorter for the Ka-band beam emitter. Furthermore, the RMSE of the velocity fluctuated slightly for the duration.

\section{Conclusions}

In this paper, a location-awareness method that uses a CubeSat was proposed as a new approach to monitor and identify the emitters of GEO communications satellites on a global scale. The proposed work represented a preliminary analysis of the problem. A realistic scenario in which a CubeSat receives signals from GEO communications satellites was simulated using the STK, through which bearing and position data were collected. The geometry, the spacecraft equations of motion, the measurement process, and the EKF equations in a location system were addressed with respect to the location methods investigated in this study. The results of the simulation and the analysis presented in this study provide a thorough verification of the location-awareness method's performance.

The bearing measurement accuracy and the initial state error are two critical parameters that impact the capability of the location-awareness method significantly. Therefore, to satisfy the performance requirement of the locationawareness method, the bearing measurement is required to be accurate to within $0.01^{\circ}$, and the initial position error is required to be less than $10^{6} \mathrm{~m}$. As a difficult case, the positon of a Ka-band beam emitter was estimated successfully to show that the method is suitable for emitters with various frequencies. Most importantly, no evidence of instability was observed in all tests. Therefore, it is concluded that the location-awareness method offers an active solution for monitoring and identifying the emitters of GEO communications satellites.

\section{Conflicts of Interest}

The authors declare that there are no conflicts of interest regarding the publication of this paper.

\section{Acknowledgments}

This work is sponsored by the Natural Science Foundation of China (Grant no. 61471021) and the China Academy of Launch Vehicle Technology Foundation. The authors would like to thank Lina Bao of Tianjin University of China for her invaluable advice.

\section{References}

[1] US Government, "National Security Space Strategy Unclassified Summary," US Policy, 2011.

[2] J. Garamone, "Shelton Discusses Importance of Space Defense," Defense News, 2014.

[3] US Government, "FCC Online Table of Frequency Allocations," Federal Communications Commission Office of Engineering and Technology Policy and Rules Division, 2014.
[4] Y. Henri, "ITU Radiomonitoring," in Proceedings of the TU AsiaPacific Regional Workshop on Satellite Launching \& Coordination, Indonesia, 2013.

[5] J. A. LaSarge and J. Black, "A cubesat mission and configuration analysis for locating and mapping spot beams of geostationary comm-satellites," in Proceedings of the 53rd AIAA Aerospace Sciences Meeting, 2015, USA, January 2015.

[6] R. Bentley, "Experimental investigation of radio frequency (RF) signal geolocation concepts using geostationary satellites," in Proceedings of the Space Programs and Technologies Conference, Huntsville, Ala, USA.

[7] A. J. Small, Radio Frequency Emitter Geolocation Using CubeSats [ms thesis], Department of Electrical and Computer Engineering, Air Force Institute of Technology (AU), Wright-Patterson AFB, Ohio, USA, 2014.

[8] V. J. Aidala, "Kalman Filter Behavior in Bearings-Only Tracking Applications," IEEE Transactions on Aerospace and Electronic Systems, vol. 15, no. 1, pp. 29-39, 1979.

[9] V. J. A. Ala and S. C. Nardone, "Biased Estimation Properties of the Pseudolinear Tracking Filter," IEEE Transactions on Aerospace and Electronic Systems, vol. 18, no. 4, pp. 432-441, 1982.

[10] S. A. Gadsden, D. Dunne, S. R. Habibi, and T. Kirubarajan, "Comparison of extended and unscented Kalman, particle, and smooth variable structure filters on a bearing-only target tracking problem," in Proceedings of the Signal and Data Processing of Small Targets 2009, San Diego, Calif, USA, August 2009.

[11] J. M. C. Clark, R. B. Vinter, and M. M. Yaqoob, "Shifted Rayleigh filter: A new algorithm for bearings-only tracking," IEEE Transactions on Aerospace and Electronic Systems, vol. 43, no. 4, pp. 1373-1384, 2007.

[12] L. Schmitt and W. Fichter, "Continuous singularity free approach to the three-dimensional bearings-only tracking problem," Journal of Guidance, Control, and Dynamics, vol. 39, no. 12, pp. 2673-2682, 2016.

[13] F. Reali and G. Palmerini, "Estimate problems for satellite clusters," in Proceedings of the IEEE Aerospace Conference (AC '08), Big Sky, Mont, USA, March 2008.

[14] S. Segan and D. Marceta, "Orbit determination and parameter estimation: extended kalman filter (EKF) versus least squares orbit determination (LSQOD)," Publications of the Astronomical Observatory of Belgrade, vol. 86, pp. 197-202, 2009.

[15] E. Glennon, J. Gauthier, M. Choudhury, K. Parkinson, and A. Dempster, "Project Biarri amd the Namuru V3.2 Spaceborne GPS Receiver," in Proceedings of the International Global Navigation Satellite Systems Society (IGNSS) Symposium, Outrigger Gold Coast, Australia, 2013.

[16] IPC BonAnza LLC, "Satbeams," http://www.satbeams.com, 2014.

[17] R. Horak, Telecommunications and Data Communications Handbook, John Wiley \& Sons, Hoboken, NJ, USA, 2012.

[18] L. Ersoy and G. Schennum, "INTELSAT VII spacecraft antennas," in Proceedings of the Digest on Antennas and Propagation Society International Symposium, Ford Aerospace, San Jose, Calif, USA, 1989.

[19] G. Schennum and L. Ersoy, "Antenna subsystem for the INTELSAT VII spacecraft," in Proceedings of the IEEE Conference on Aerospace Applications, Ford Aerospace, Space Systems Division, Vail, Colo, USA.

[20] D. Roddy, Satellite Communications, McGraw-Hill Companies, New York, NY, USA, 4th edition, 2006. 
[21] M. Pontani and P. Teofilatto, "Satellite constellations for continuous and early warning observation: A correlation-based approach," Journal of Guidance, Control, and Dynamics, vol. 30, no. 4, pp. 910-920, 2007.

[22] B. Friedlander and A. J. Weiss, "Direction Finding for WideBand Signals Using an Interpolated Array," IEEE Transactions on Signal Processing, vol. 41, no. 4, pp. 1618-1634, 1993. 


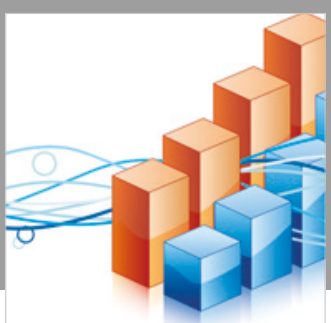

Advances in

Operations Research

\section{-n-m}
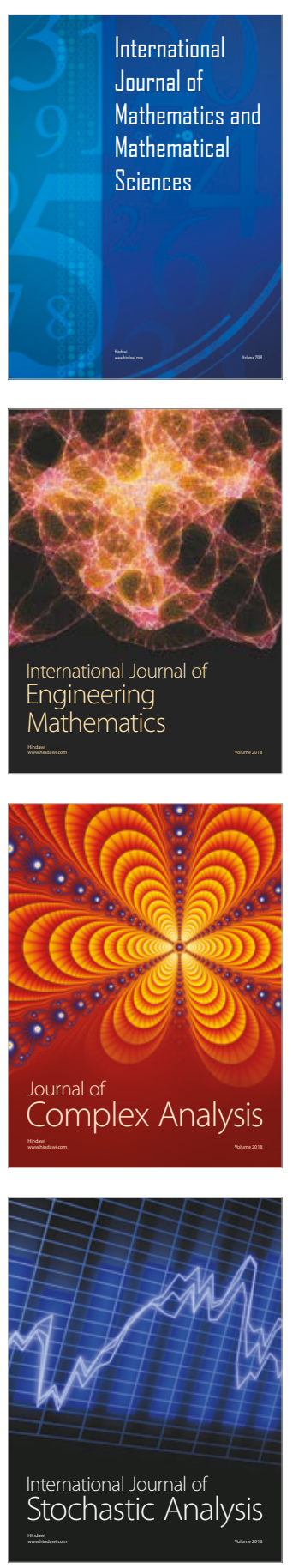
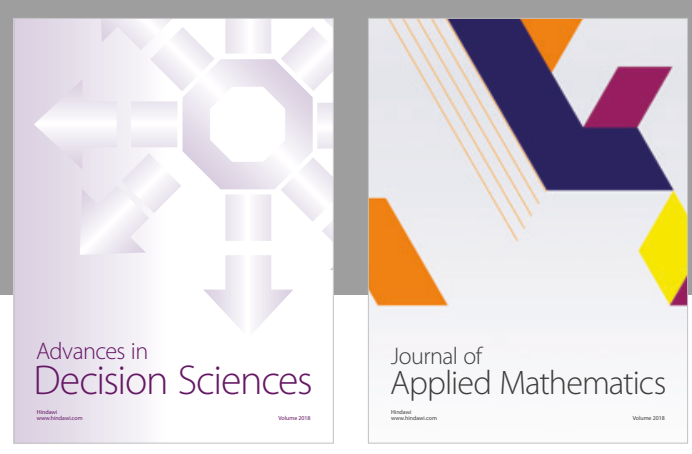

Journal of

Applied Mathematics
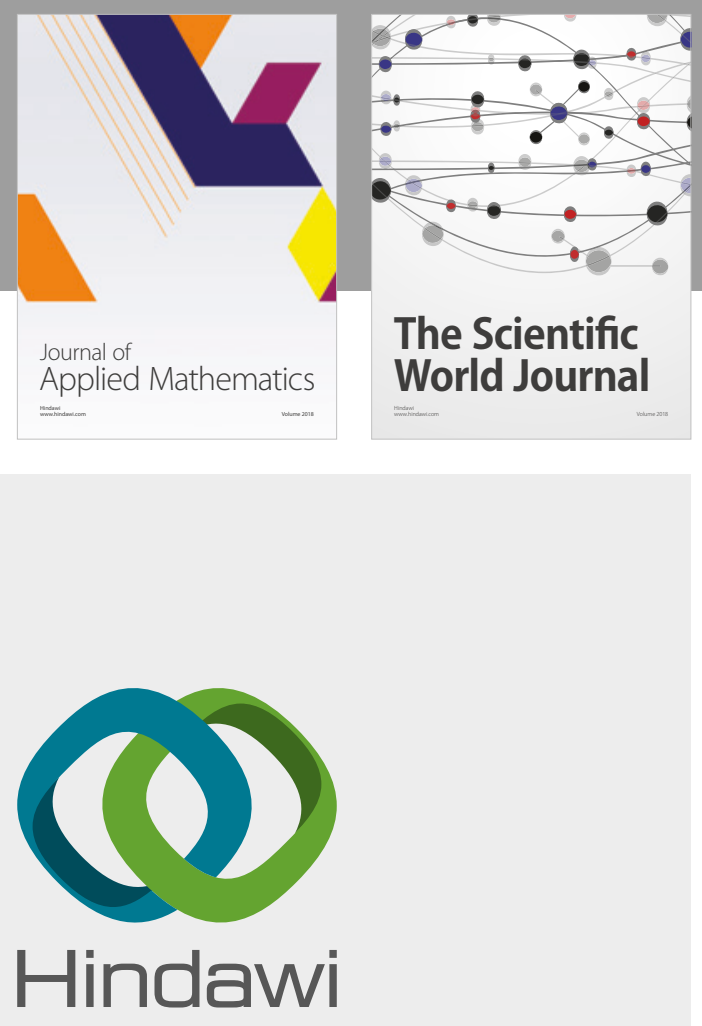

Submit your manuscripts at

www.hindawi.com

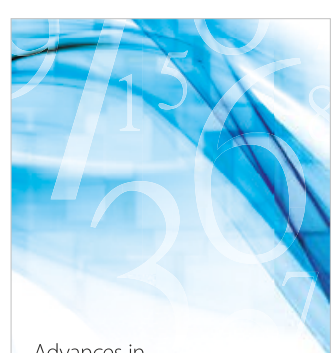

Advances in
Numerical Analysis
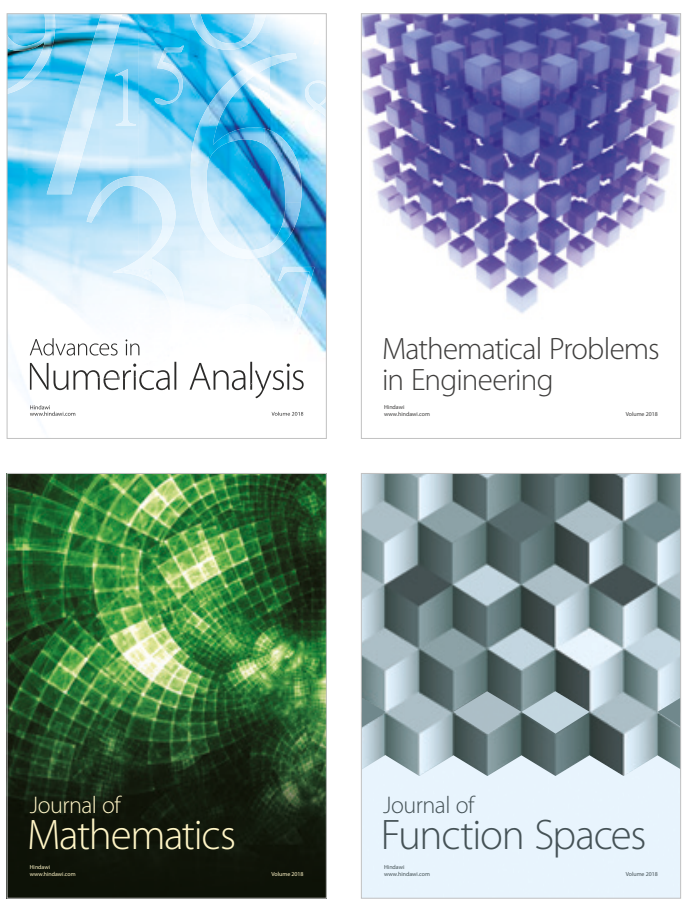

Mathematical Problems in Engineering

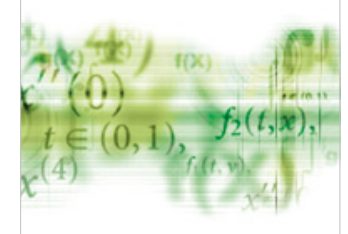

International Journal of

Differential Equations

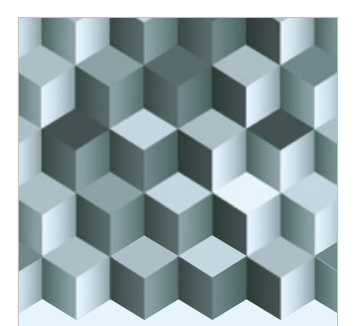

Journal of

Function Spaces

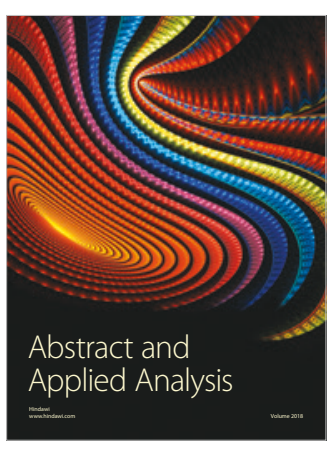

The Scientific

World Journal

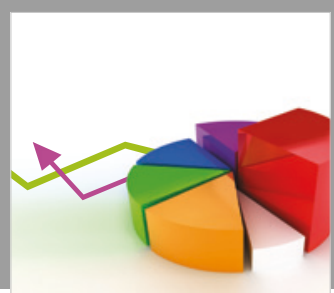

Journal of

Probability and Statistics
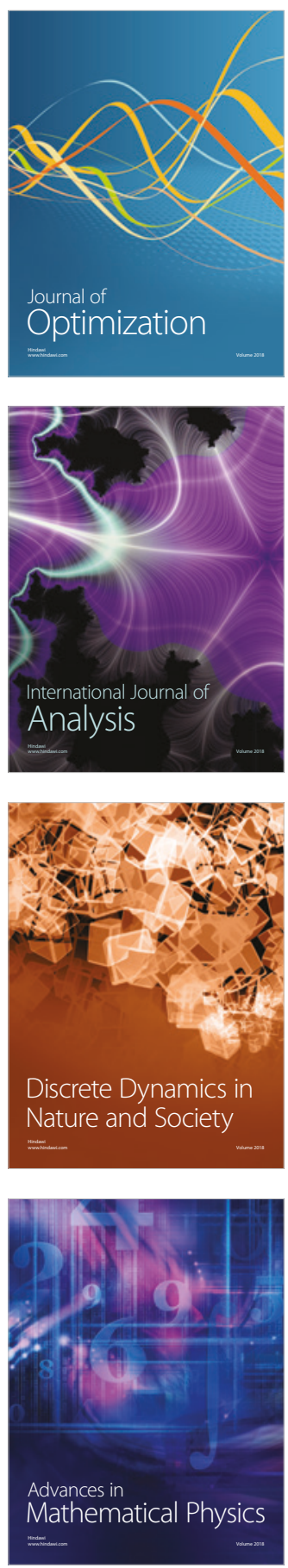